\title{
Urgences
}

\section{Reflet des États-Unis dans le roman québécois : une version de l'Amérique}

\section{Jean-François Chassay}

Numéro 34, décembre 1991

Mythes et Romans de l'Amérique

URI : https://id.erudit.org/iderudit/025681ar

DOI : https://doi.org/10.7202/025681ar

Aller au sommaire du numéro

Éditeur(s)

Urgences

ISSN

0226-9554 (imprimé)

1927-3924 (numérique)

Découvrir la revue

Citer cet article

Chassay, J.-F. (1991). Reflet des États-Unis dans le roman québécois : une version de l'Amérique. Urgences, (34). https://doi.org/10.7202/025681ar d'utilisation que vous pouvez consulter en ligne.

https://apropos.erudit.org/fr/usagers/politique-dutilisation/ 


\section{Reflet des États-Unis dans le roman québécois: une version de l'Amérique}

Jean-François Chassay

Dans Le royaume de Morphée 1 , le jeune Carl Hausman assiste à un match de baseball et voit une balle disparaittre dans un bosquet. Il part à sa recherche et pénètre dans une grotte où se révèle à lui un univers féérique, amorçant un parcours qui lui fait redécouvrir de nombreux mythes littéraires de la culture occidentale. L'ombre de Dante passe ici en silence.

Comment interpréter ce passage obligé par le baseball (qui ne dure que quelques pages)? Le royaume de Morphée, écrit par un New-Yorkais, ne répond manifestement pas aux canons habituels du roman américain. Télescopage artificiel qui ne vise qu'à entretenir la confusion? Volonté arbitraire d'entretenir au départ l'illusion référentielle pour mettre davantage en évidence l'effraction du réel par le merveilleux? Peut-être tout simplement (mais, justement, il n'y a peut-être là rien de simple), marquer ironiquement le lieu d'où l'on parle en inscrivant textuellement l'Amérique dans le texte, son Amérique.

On pourrait imaginer ce roman écrit par un auteur du Sud (Borgès n'est pas loin) mais 3 pages sur 420 inscrivent "l'américanité" de son auteur. Toute la mécanique textuelle découle de là: pas de retour aux sources de la modernité pour Steven Millhauser sans le baseball, c'est-à-dire sans les ÉtatsUnis et ses références culturelles. Le parcours initiatique que devra affronter le jeune Carl Hausman doit nécessairement prendre sa source à cet endroit.

L'anecdotique permet parfois de poser, sinon de désamorcer, un problème conceptuel de taille. Si la notion «d'américanité " conduit à tant de généralités (et à de nombreuses éruptions cutanées chez les gens qui possèdent un peu de rigueur), c'est parce qu'on ne résume pas en deux coups de cuiller à pot une notion culturelle et littéraire englobant un 
territoire (et les sociétés qui y vivent) qui s'étend du cap Horn au Groenland, couvrant 42 millions de kilomètres carrés, peuplé de plus de 700 millions d'habitants parlant quatre langues officielles (soyons narcissique et incluons le français, malgré sa sous-représentation par rapport aux trois autres). On chercherait en vain une définition idoine à un phénomène d'une telle ampleur.

* L'américanité * se manifesterait d'abord comme un symptôme, celui qui permet éventuellement de révéler le lieu d'où l'on parle. Donner sens à l'espace culturel, comme s'il fallait toujours le définir et prouver son existence. Quitte à rappeler des faits qui risquent de passer aux yeux de certains pour des évidences, il faut répéter que «le dénominateur commun des littératures des Amériques, y compris la québécoise, réside dans le fait que, dès leur naissance, elles se nourrissent [...] de la culture européenne * ${ }^{2}$, et que parler de «la totalité de l'Amérique», c'est considérer dans son ensemble une « terre de conquête et de métissage où l'Europe est venue organiser horizontalement un territoire vertical " ${ }^{3}$. A partir de ces affirmations, la question de l'identité s'impose au cour de ces littératures. On a tellement insisté pour dire que l'Amérique vivait sans Histoire $^{4}$ qu'on a réussi à mettre en évidence, presque sans le vouloir, l'importance fondamentale de celle-ci dans la fiction.

Après avoir dit cela, nous ne sortons pas pour autant du domaine des généralités, et pour cause: cette amorce de définition ne peut masquer les immenses lacunes du commentateur par rapport à l'ensemble du corpus. Toute tentative pour affirmer l'existence et le sens d'un concept comme "l'américanité" s'effectue à partir d'une connaissance

\footnotetext{
2 Zila Bernd, \& La quête d'identité: une aventure ambiguë ", Voix \& images, 34 (dossier * Québec-Amérique latine "), automne 1986, p. 21.

3 Gilles Thérien, * La littérature québécoise, une littérature du tiersMonde?", Voix \& images, ibid., p. 14.

4 Encore dernièrement, à propos d'un roman de l'Américain Richard Ford (Une saison ardente), on citait Salman Rushdie affirmant voir dans ce texte “Une Amérique privée d'Histoire" (Patrick Raynal, Le monde, vendredi 3 mal 1991, p. 20). Parler d'absence d'Histoire à propos d'un corpus romanesque où elle est depuis plusieurs décennies omniprésente (même si elle est souvent présentée par un biais négatí) confine au cliché et l'enferme dens le vieux mythe de la nature qui serait la seule échappatoire de la littérature américaine.
} 
parcellaire à la fois des littératures concernées mais aussi de l'Histoire, à moins de vouloir être radicalement réducteur. S'aventurer à affirmer par exemple, pour l'ensemble de la littérature des Amériques, que «la réalité culturelle étrangère [du colonisateur] est perçue comme supérieure à la culture nationale * 5 est un peu court. La déclaration apparaît douteuse quant aux rapports entretenus entre le Brésil et le Portugal ou entre les Etats-Unis et la Grande-Bretagne. Déclarer que "ce sera sous le signe de l'hybridisation que vont se construire les littératures des Amériques " ${ }^{6}$, formule séduisante et opératoire pour certains corpus nationaux, c'est ne pas tenir compte en réalité d'une grande partie de la littérature des États-Unis, et non la moindre. Catapulté par ailleurs, grâce à certains commentateurs, au rang de mot fétiche du vocabulaire québécois, le terme "américanité " n’a pas suffi à masquer la vacuité du discours de ceux qui n'avaient déjà rien à dire. Un programme comme celui qui consiste à « relever le défi d'écrire et de parler un langage neuf sur un continent neuf " ${ }^{7}$ rend peut-être compte d'un sympathique enthousiasme et d'une diligente volonté de s'exprimer mais apporte peu à la réflexion et signale moins une appartenance (qui manifesterait un sentiment d'identification au moins relativement commun à ceux qui participent de cette "américanitén) qu'une volonté d'originalité dont on chercherait en vain chez l'auteur cité les tenants et les aboutissants.

En réalité, après avoir fait un usage compulsif de ce substantif au début de la dernière décennie, on a pris conscience de l'imprécision à laquelle conduisait son emploi: doute sur la possibilité d'une véritable définition ${ }^{8}$, risque de tomber dans

5 Zila Bernd, "La quête d'identité: une aventure ambiguë ", Voix \& images, op. cit., p. 21.

6 Ibid.

7 Claude Beausoleil, Les livres parlent, coll. "Estacades", Trois-Rivières, Écrits des forges, 1984, p. 28. Ce livre, comme plusieurs articles et ouvrages mentionnés dans cet article, a été retracé grâce à la très précieuse bibliographie de Benoît Melançon. Voir \& La littérature québécoise et l'Amérique. Prolégomènes et bibliographie ", Études françaises, vol. XXVI, $n^{\circ} 2$ (numéro intitulé L'Amérique de la littérature québécoise), automne 1990, p. 65-108.

8. Lucie Robert, « L'américanité de la dramaturgie québécoise ", Études françaises, vol. XXVI, $n^{\circ} 2$ (numéro intitulé L'Amérique de la littérature québécoise), autornne 1990, p. 62. 
la pure mythification ${ }^{9}$ ou la tautologie, la littérature québécoise devant être considérée d'office comme américaine puisqu'elle s'écrit en Amérique ${ }^{10}$. Peut-être faut-il finalement considérer l'Amérique, à la suite de Réjean Beaudoin, comme un éternel * projet [dont] l'image de son devenir reste l'épreuve incessante de son recommencement ${ }^{11}$. Symptomatiquement, le Festival de Théâtre des Amériques, présenté à Montréal et consacré lors de ses deux premières éditions à des pièces novatrices provenant des trois Amériques, s'est ensuite ouvert aux spectacles européens. D'une définition territoriale, le mot *Amérique est passé au symbolique: mythe de la nouveauté et de la découverte.

Cet «éternel projet» prend pourtant son sens sur un territoire et dans des contextes socio-culturels singuliers. L'examen de la notion d'américanité, malgré ses nombreuses omissions et insuffisances jusqu'à maintenant, a au moins eu pour effet d'élargir le champ des intérêts littéraires québécois et d'ajouter au traditionnel axe est-ouest un axe nord-sud ${ }^{12}$. Les obstacles que doit affronter le chercheur qui tente d'en arriver à une définition totalisante (est-elle vraiment nécessaire?) de ce qu'on nomme confusément *américanité * ne doit pas pour autant restreindre cette approche continentale du phénomène littéraire, au contraire. La lecture comparatiste, dans une perspective continentale, peut permettre de mieux cerner et comprendre l'espace social et culturel tel qu'il s'exprime dans la littérature, dans la mesure où l'analyse le prend en charge.

Toutes ces littératures [américaines] dont certaines sont à la pointe de ce qui se fait (en roman par exemple) n'ont-elles pas connu des problèmes analogues de colonisation, de

9 Bernard Andrès, * La littérature québécoise à Voix \& images: créneau ou ghetto? ", Voix \& images, 35 (dossier intitulé * La question des revues"), hiver 1987, p. 309-310.

10 Benoît Melançon, « La littérature québécoise et l'Amérique. Prolégomènes et bibliographie ", op. cit., p. 72.

11 Réjean Beaudoin, "Rapport Québec-Amérique ", Possibles, vol. VIII, n० 4 (numéro intitulé L'Amérique inavouable), été 1984, p. 46.

12 Bien qu'il faille nuancer: cet axe est-ouest se limite bien souvent à la France et d'abord pour des raisons institutionnelles avant d'être littéraires. Est-il plus souvent question de la littérature britannique ou allemande que mexicaine ou brésilienne, dans les journaux, magazines et revues québécois? 
post-colonisation, de rapports aux modes et aux codes européens? C'est là tout un programme que nous commençons à peine à entrevoir. Les problèmes d'épistémologie comparatiste doivent être posés dans une perspective québécoise. ${ }^{13}$

La dernière phrase est essentielle: elle indique la nécessité d'un point d'ancrage sans lequel ce qui précède pourrait conduire à de nouvelles généralités. Il ne s'agit pas de renier * le relais européen *, pour reprendre une expression de François Ricard ${ }^{14}$, mais d'éventuellement le retrouver en passant par le biais du relais américain.

\section{Les États-Unis lus du Québec}

Lecteur depuis plusieurs années à la fois de romans américains («états-uniens») et de romans québécois, j’en suis arrivé, par une pente naturelle en quelque sorte, à chercher les traces de la présence américaine dans le roman québécois. Ceci ne va pas de soi a priori dans la mesure où l'influence explicite de la littérature américaine a relativement peu d'incidences directes sur le corpus québécois contemporain. C'est pourquoi on se contente souvent de considérer les déplacements transcontinentaux, dans le roman québécois, comme un signe de l'influence américaine, ceci au détriment de l'analyse discursive. Comme tous les clichés, celui qui consiste à situer le roman des Amériques à l'intérieur d'une poétique de l'espace possède un fond de vérité mais conduit assez rapidement à un cul-de-sac ${ }^{15}$. Comme l'écrit Benoît Melançon, «l'Amérique n'est que rarement un texte [dans la littérature québécoise]; plus souvent elle est un territoire " 16 . Plusieurs

13 Bernard Andrès, « La littérature québécoise à Voix \& images: créneau ou ghetto? ", op. cit., p. 310.

14 François Ricard, "Le relais européen " dans La littérature contre elleméme, Montréal, Boréal express, 1985, p. 187-191.

15 Cela étant dit, il serait intéressant d'analyser les déplacements spatiotemporels dans le roman québéccis depuis le XIX siècle. Le roman qui suit la Deuxième Guerre, de Pierre Baillargeon à Pierre de Grandpré, favorise les déplacements est-ouest, la France restant la grande référence culturelle. II est possible que les années 1945-1970 aient été les seules où la France, comme lieu physique, a eu plus d'importance que les États-Unis dans le roman québécois.

16 Benoît Melançon, * La littérature québécoise et l'Amérique. Prolégomènes et bibliographie", op. cit., p. 71 . 
raisons pourtant justifient cette lecture du roman québécois à travers le prisme des États-Unis.

Depuis toujours, le Québec entretient avec les États-Unis des rapports conflictuels ${ }^{17}$ dont le roman peut rendre compte adéquatement en tant qu'espace de tension à l'intérieur duquel se manifeste le discours d'une société. Cette définition tend à se superposer à celle déjà avancée par Claude Duchet:

Ce que je propose d'appeler discours social n'est pas propre au roman mais se manifeste dans le roman d'une manière spécifique dans la mesure où celui-ci, fonctionnant comme une société, reproduit dans son texte un ensemble de voix brouillées, anonymes, une sorte de fond sonore - ce que $M$. Foucault nomme l'arrière-fable - où se mêlent les clichés, les fameuses idées reçues, les stéréotypes socio-culturels, les idiolectes caractérisants, les traces d'un savoir institutionnalisé ou ritualisé, des noyaux ou fragments d'idéologies plus ou moins structurés, plus ou moins subsumés par une idéologie dominante, plus ou moins actualisés par des références, inscrits dans des lieux comme dans des personnages, voire montés en scènes ou éléments de scènes. ${ }^{18}$

Autrement dit, le roman fait surgir des «lignes de force, celles-là mêmes que les pressions de l'actualité ne permettent pas de distinguer "19. Dans cette perspective, il ne s'agit pas de comparer des œuvres entre elles mais plutôt de voir comment l'imaginaire romanesque au Québec révèle la présence américaine et les orientations sociales, les positions idéologiques ou les contradictions qu'elle provoque. Une épisté-

17 Voir à ce sujet, de Guildo Rousseau, L'image des États-Unis dans la littérature québécoise (1775-1930), Sherbrooke, Naaman, 1981, 356 p. Pour analyser les tensions entre le Québec et les États-Unis dans le roman québécois plus récent, on pourra lire mon texte intitulé "L'autre ville américaine " a paraitre en 1992 dans un ouvrage collectif du groupe de recherche "Montréal imaginaire " du Département d'études françaises de l'Université de Montréal intitulé Montróal à travers les áges. On en trouvera un extrait, dans une forme légèrement différente, sous le titre * Montréal et les États-Unis: idéologie et interdiscursivité chez Jean Basile et Réjean Ducharme ", Voix \& images, 48, printemps 1991, p. 503-513.

18 Claude Duchet, "Discours social et texte italique dans Madame Bovary", Lettres modernes, 32 (numéro intitulé Langages de Flaubert), 1976, p. 145.

19 Gilles Marcotte, introduction à Littérature et circonstance, Montréal, L'Hexagone, 1989, p. 10. 
mologie comparatiste viserait à montrer comment, dans le roman, l'image du Québec prend forme en se situant dans la mouvance culturelle américaine et en résistant à celle-ci, en refusant de se laisser absorber. Lié aux États-Unis politiquement, économiquement, socio-culturellement, le Québec n'a jamais pu faire abstraction du discours américain. « Ce n'est pas sous le mode du projet mais bien sous celui plus subtil et silencieux de l'espace, de l'environnement urbain et de la quotidienneté, que l'américanitude est vécue dans la culture québécoise. " ${ }^{20}$ Que l'on considère cet *envahissement * comme une contrainte épouvantable ou une chance inestimable, son analyse sur le plan littéraire n'en apparaît pas moins nécessaire.

L'image projetée des États-Unis au Québec provient souvent de France: des essais comme ceux de Jean Baudrillard ${ }^{21}$ et celui de Léo Sauvage ${ }^{22}$ naviguent entre naïveté et sentiment d'étrangeté et on ne saurait vraiment s'y reconnaître. Les traductions de romans américains en France laissent parfois un arrière-goût désagréable. Il serait d'ailleurs intéressant d'analyser un corpus de traduction dans l'optique où Annie Brisset a étudié celui du théâtre au Québec ${ }^{23}$ et de voir comment les textes ont parfois été «francisés" pour les besoins du public hexagonal ${ }^{24}$. Il serait vain et mesquin, ceci

20 Raymond Montpetit, "Culture et milieu de vie: l'espace urbain à Montréal ', Écrits du Canada français, 58 (numéro intitulé Québec/USA), 1986, p. 132.

21 Jean Baudrillard, Amérique, Paris, Grasset, 1986, 249 p.; de nombreux fragments de Cool Memories I, Paris, Galilée, 1987 et Cool Memories II, Paris, Galilée, 1990.

22 Léo Sauvage, Les Américains, Paris, Mazarine, 1983, 766 p.

23 Annie Brisset, Sociocritique de la traduction, Montréal, Le Préambule, 1990, $347 \mathrm{p}$. Elle montre comment, très souvent, la traduction québécoise a " récupéré" le texte étranger, soit sur le plan institutionnel, soit en l'adaptant pour en faire une version « nationale ".

24 La préface célèbre de Valéry Larbaud à Tandis que j'agonise de Faulkner est de ce point de vue éclairante. L'écrivain félicite le traducteur Maurice Edgar Coindreau qui « a fait sagement en ne cherchant pas à rendre les caractéristiques du patois que parlent les personnages de Tandis que j'agonise. Ce patois peut être curieux pour le lecteur de langue anglaise [...] mais ce n'est guère qu'un anglais dégradé, entaché de négligence et de mauvaises habitudes, qui nous a paru plus difficile que savoureux, bien qu'il reflète les conditions d'existence de ceux qui le parlent et soit bien dans l'atmosphère du livre. " William Faulkner, Tandis que j'agonise, coll. « Folio ", Paris, Gallimard, 
dit, de critiquer ce que les Français disent parfois des ÉtatsUnis. Leurs critères ne sont pas nécessairement les nôtres et leur point de vue n'a pas à être jugé à l'aune du Québec. Mais c'est une raison supplémentaire pour chercher à articuler un discours cohérent sur les États-Unis plutôt que de s'irriter de ce qui vient d'ailleurs.

Dans *Le relais européen *, François Ricard, évoquant à la fois l'importance de l'Europe et des États-Unis pour le Québec ( Quoi qu'il en soit, ces deux relais existent, je les utilise l'un et l'autre et il serait vain de vouloir éliminer celui-ci en faveur de celui-là, vain et dangereux. " ${ }^{25}$ ) privilégie la première: * aller plutôt du côté de l'Europe et du relais français, c'est véritablement agir, c'est-à-dire tenter de résister à une détermination et travailler à l'instauration d'un état de choses qui n'aille pas nécessairement de soi " 26 , alors qu'aller du côté du relais américain ne consiste qu'à entériner ce qui se trouve déjà là:

Le contexte socio-culturel où je vis, de même que le contexte économique, n'est pas seulement influencé par les USA, littéralement il leur appartient, il est structuré et déterminé par eux dans presque toutes ses dimensions, mais surtout dans certaines des plus fondamentales, comme la culture de masse et les communications. II y a une sorte de loi de pesanteur qui joue ici en faveur des USA, si bien que la moindre inertie, le moindre relâchement de ma part augmente automatiquement mon insertion dans le réseau américain. 27

Renversons la perspective pour conclure autrement: parce que la présence américaine ne repose pas sur des spéculations mais au contraire sur des situations on ne peut plus concrètes, il s'avère d'autant plus important de chercher à débusquer les idéologèmes qui se rattachent à la présence des États-Unis dans le champ socio-culturel, de voir comment on y résiste ou comment on s'y soumet. Le passé français, plus lointain, a aujourd'hui des déterminations plus « abstraites " écrit François Ricard, essentiellement linguistiques. Or, étudier le

1973, p. 11). La " pureté de la langue " justifie qu'on fasse dire au texte ce qu'on a envie qu'il dise. Les intentions de l'auteur pèsent peu, même quand il se nornme William Faulkner.

25 François Ricard, "Le relais européen ", op. cit., p. 188.

26 lbid., p. 189.

27 lbid., p. 188. 
roman québécois dans une perspective culturelle axée sur l'influence américaine, évite de réduire encore une fois la question de la spécificité culturelle à la langue. Comment, face à un voisin aussi étouffant, ayant une personnalité aussi forte, le roman québécois contemporain peut-il inventer sa propre Amérique? Voilà selon moi une des questions essentielles à poser aujourd'hui à propos de la littérature québécoise.

\section{L'idéologie de la communication: hypothèse de travail}

L'Histoire a servi de point de départ à Guildo Rousseau pour son livre sur L'image des États-Unis dans la littérature québécoise. L'ajout des dates (1775-1930) indique l'évolution chronologique privilégiée dans cet ouvrage. Mais à cette perspective linéaire où l'Histoire est retracée de manière événementielle grâce à un réseau de thèmes assez lâche, on peut en substituer une autre pour l'époque contemporaine. Elle servirait à une nouvelle lecture d'une partie du corpus romanesque québécois dans un axe américain et privilégierait une approche méthodologique qui tiendrait compte de ce qu'on a nommé * l'idéologie de la communication *.

L'histoire contemporaine n'est pas seulement celle qui prend en vue les années qui nous sont chronologiquement les plus proches; plus rigoureusement, elle est l'histoire de cette époque où, par le biais de l'utilisation de nouveaux moyens de communication (la télévision en particulier), tout tend à s'écraser sur le plan de la contemporanéité et de la simultanéité, produisant ainsi une dés-historicisation de l'expérience. 28

Ce risque d'un aplatissement de la dimension historique se situe dans le cadre d'une société dominée par l'idéologie de la communication, « discours explicite et cohérent centré sur le thème de la communication [...] apparu au milieu du siècle [...], véritable alternative aux idéologies politiques perçues à l'époque dans ces milieux comme ayant fait faillite dans la gestion des affaires humaines" ${ }^{29}$. Si celle-ci couvre mainte-

28 Gianni Vattimo, La fin de la modernité, Paris, Seuil, 1987, p. 16.

29 Philippe Breton et Serge Proulx, L'explosion de la communication, Paris et Montréal, La Découverte et Boréal, 1989, p. 11-12. 
nant l'Occident, elle a pris naissance en Amérique du Nord, dans une société * sans mémoire". Dans ce contexte, la communication serait apparue comme * le recours d'une collectivité pauvre en symboles historiques $* 30$.

Cette vision circulaire de l'Histoire conduit également à une fragmentation de l'espace et des discours ainsi qu'à un télescopage entre l'expérience empirique du réel et les langages des mass médias. La *guerre du faux ", pour reprendre l'expression d'Umberto Eco, devient un spectacle permanent. Ceci conduit Gianni Vattimo à affirmer que le monde actuel correspond à « une réalité "allégée" car moins nettement divisée entre le vrai et la fiction, l'information ou l'image: monde de la médiatisation totale où nous nous situons déjà pour une bonne part ${ }^{31}$.

Une tendance importante du roman américain contemporain reflète cette nouvelle réalité sociale ${ }^{32}$. À l'ère des médias électroniques, alors que le mot cybernétique fait maintenant partie du vocabulaire courant, la réalité se cache sous les masques d'une information qui se fait de plus en plus confuse, ambiguë, quand elle n'est pas carrément mensongère. Nous ne sommes plus dans le village global de Marshall McLuhan, mais bien dans le "Global Shopping Center * ${ }^{33}$ où la connaissance s'amasse pêle-mêle comme dans un gigantesque bazar, fatras des discours dans lequel chacun prend ce qu'il veut puisqu'on "peut considérer le monde comme une myriade de messages à toutes fins utiles » 34 .

L'écrivain peut exprimer cet éclatement du réel à travers la dispersion des discours en privilégiant une position critique, mais l'effet de séduction des mass médias le place aussi à la lisière de la fascination. Le danger du mimétisme

30 Lucien Sfez, Critique de la communication, Paris, Seuil, 1988, p. 139.

31 Gianni Vattimo, La fin de la modemité, op. cit., p. 185.

32 Pour plus de détails sur ce sujet, on consultera l'ouvrage de Marc Chénetier, Au-delà du soupçon. La nouvelle fiction américaine de 1960 à nos jours, Paris, Seuil, 1989, 453 p.

33 L'expression est du sociologue Todd Giltin dans * Hip-deep in PostModernism ", The New York Times Book Review, 6 novembre 1988, p. 35. On pourra aussi lire, du même auteur, une critique de la société * postmoderne" à travers l'utilisation aux États-Unis du médium télévisuel dans Inside Prime Time, New York, Pantheon, 1989, 252 p.

34 Norbert Wiener, cité par Paul Watzlawick, La réalité de la réalité, coll. «Points ", Paris, Souil, 1978, p. 13. 
est alors flagrant. En cherchant à reproduire la * culture en mosaique * de l'individu moderne, il ne va pas de soi pour autant qu'on puisse rendre compte facilement du débordement de sens propre à la réalité complexe du discours social actuel, qui renvoie - notamment par le biais du discours technologique - à des contextes de décodage plus larges que celui qui mettrait directement en relation un émetteur et un récepteur. Gerald Graff n'a pas tort, dans Literature against itself, d'affirmer que c'est justement le jeu du capitalisme moderne de * détruire tous vestiges de la tradition, toute idéologie orthodoxe, toutes formes continues et stables du réel afin de stimuler des niveaux plus élevés de consommation ${ }^{35}$.

À une époque où il semble acquis, suite aux discours scientifiques des dernières décennies, qu'il n'existe pas de réalité absolue mais seulement des conceptions subjectives et parfois contradictoires de la réalité (ou, pour citer Nietzsche annonçant la déroute des positivistes, qu'il n'y a pas de faits, rien que des interprétations), rendre compte de la réalité sociale ne va pas de soi puisque la constellation des informations provoque une consternation: l'impossibilité de contextualiser et de poser un regard englobant sur un phénomène. Pour garder une position critique, il ne suffit pas d'utiliser le concept de dispersion dans le roman mais il faut aussi penser celui-ci. L'hétérogénéité du discours dans le roman ne peut avoir un sens que s'il incite à la réflexion sur le mouvement actuel de décomposition et de recomposition autour des techniques de communication et de leur usage.

Si peu d'écrivains québécois portent autant d'intérêt à ces questions (et ont l'envergure pour le faire!) que des romanciers comme Coover, Pynchon, Barth ou McElroy, il reste qu'ils écrivent dans un contexte social fortement similaire. L'univers américain est un univers médiatique. Aux ÉtatsUnis, où les actes fondateurs du pays consistaient en réalité à se * purifier* de l'Europe, à refuser ses traditions et son histoire, le mythe a toujours été plus fort que la réalité et cette fiction a rejoint le Québec rapidement.

Raz-de-marée provenant des États-Unis, lidéologie de la communication vient frapper de plein fouet le sujet québécois

35 Cité par Marc Chénetier, Au-delà du soupçon..., op. cit., p. 77. 
en quête d'identité. Vu dans cette perspective, le rapport schizoïde au monde américain qui marque le roman québécois depuis toujours (fascination/répulsion, identification/rejet) aurait subi un glissement et concernerait plus spécifiquement aujourd'hui l'univers des communications. Celui-ci envelopperait le champ de la conscience au point qu'il ne serait plus possible de penser en-dehors de cette problématique, selon Baudrillard 36.

A travers l'idéologie de la communication, la science et la technique permettent une augmentation de l'information sous toutes ses formes: type, quantité, qualité. Comment ces discours hétérogènes (publicitaires, télévisuels; politiques, etc.), sont-ils perçus, récupérés, assimilés par le roman québécois? Alexandre Chenevert de Gabrielle Roy, publié en 1954, apparaît dans cet esprit comme un roman fondateur, puisque le personnage éponyme incarne spécifiquement le citadin aliéné par le discours de l'information ${ }^{37}$.

Au cours des quarante dernières années, comme au cours des décennies précédentes, une partie importante du corpus romanesque québécois s'est inscrite dans l'orbe de la culture américaine. Il ne pouvait en être autrement. Cependant, le développement des théories des communications et l'idéologie $y$ afférant stimule une lecture des textes qui répond moins à une thématique (comme chez Guildo Rousseau) qu'à une analyse discursive qui permettrait de voir comment s'exprime la pensée américaine dans un contexte québécois et comment l'imaginaire romanesque y réagit. Ces propositions de recherches débordent le cadre littéraire et s'intéressent en fait aux rapports complexes entre roman et culture ainsi qu'aux problèmes d'identité culturelle en se situant dans la perspective de la nord-américanité de la production romanesque québécoise.

L'espace manque ici pour donner des exemples tangibles du type d'analyse que permettrait cette approche théorique. On peut déjà imaginer que de nombreux sujets mériteraient d'être abordés selon cette optique: l'isotopie de la frontière,

36 Cité par Philippe Breton et Serge Proulx, L'explosion de la communication, op. cit., p. 240.

37 On pourra lire à ce sujet mon texte a paraitre dans Montréal à travers les áges, op. cit. 
l'univers urbain (Montréal et les Montréalais face à New York, San Francisco), l'intertexte américain, la légende qu'on a forgée autour de Jack Kerouac, le mythe de la Californie, le concept de territoire, la langue, etc.

Il ne s'agit pas de toute l'Amérique mais uniquement d'une de ses versions, celle qui nous est la plus proche géographiquement et culturellement. Ce n'est pas plus simple pour autant et il serait temps qu'on y accorde un peu plus d'attention. 